\title{
SÍNTESE DA PENEIRA MOLECULAR MCM-41 E SUA UTILIZAÇÃO COMO ADSORVENTE NA REMOÇÃO DO CORANTE AZUL REATIVO BF-5G
}

\author{
L. A. LIMA ${ }^{1 *}$, M. F. MOTA ${ }^{1}$, V. M. R. MENEZES ${ }^{1}$, J. R. SCHEIBLER ${ }^{1}$, M. G. F. RODRIGUES ${ }^{1}$ \\ ${ }^{1}$ Universidade Federal de Campina Grande, Departamento de Engenharia Química. \\ *liliandradelima@yahoo.com.br
}

\begin{abstract}
RESUMO - O destino de efluentes industriais representa grandes preocupações para a preservação ambiental. A indústria têxtil é responsável por gerar grandes quantidades de efluentes de composição extremamente heterogênea, com quantidade de material tóxico recalcitrante, o que torna seu tratamento mais difícil. Este trabalho teve como objetivo sintetizar a peneira molecular MCM-41 e avaliar seu potencial como adsorvente na remoção do corante azul reativo. A MCM-41 foi sintetizada utilizando o tratamento hidrotérmico, por um período de 7 dias. O material obtido foi caracterizado por Difração de Raios X, Adsorção física de $\mathrm{N}_{2}$. No estudo da remoção do corante foi aplicado um planejamento experimental do tipo fatorial $2^{2}$ com ponto central, visando avaliar a influência da quantidade de adsorvente e do $\mathrm{pH}$ da solução modelo no processo de remoção. A MCM-41 apresentou melhores resultados na remoção do corante quando combinados níveis superior e inferior para quantidade de adsorvente e $\mathrm{pH}$.
\end{abstract}

\section{INTRODUÇÃO}

O destino de efluentes industriais representa, atualmente, uma das grandes preocupações para a preservação do meio ambiente e da vida humana (Chaves 2011). Por falta de conscientização e de estação de tratamento dos resíduos, muitas indústrias optam por despejá-los em esgotos, terrenos baldios, lagos e rios. Um fator preocupante reside no fato dos resíduos se acumularem, espontaneamente, em corpos d'água, uma vez que o próprio ciclo hídrico do planeta contribui para isso, lavando ar e solo periodicamente (Oliveira et al., 2012).

A remoção de corantes dos efluentes é um dos grandes problemas enfrentados pelo setor têxtil. A elevada estabilidade biológica dos corantes dificulta sua degradação pelos sistemas de tratamento convencionais (normalmente lodo ativado) empregados pelas indústrias têxteis. A contaminação de rios e lagos com estes compostos provocam, além da poluição visual, sérios danos à fauna e flora destes locais. Com suas intensas colorações, os corantes restringem a passagem de radiação solar, diminuindo a atividade fotossintética natural, provocando alterações na biota aquática e causando toxicidade aguda e crônica destes ecossistemas (Guarantini e Zanoni, 2000). 


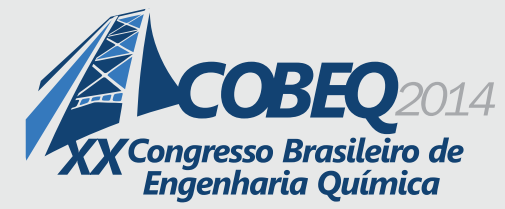

Várias técnicas têm sido aplicadas para separar os corantes da água, entre elas podemos citar os processos de adsorção, que consiste na transferência de um ou mais constituintes da fase líquida ou gasosa para a superfície da fase sólida (Vimonses et al., 2009). Materiais como, cinzas de casca de arroz, argila, zeólitas e outras peneiras moleculares foram citadas como materiais adsorventes na literatura (Oliveira et al., 2012). Contudo, a avaliação de novos materiais na remoção de corantes tem enorme significância.

Os materiais mesoporosos do tipo MCM-41 são conhecidos pela sua peculiar estrutura porosa altamente ordenada com arranjo hexagonal de poros uniformes que lhe confere elevada área superficial específica entre 1000 e $1200 \mathrm{~m}^{2} / \mathrm{g}$, dimensões adaptadas de canal entre 1,5 e 10 nm (Ryoo et al, 1998).

Este estudo foi realizado para sintetizar e caracterizar a peneira molecular MCM-41, visando seu potencial como adsorvente para remoção do corante Azul reativo BF-5G.

\section{METODOLOGIA}

\subsection{Síntese da Peneira Molecular MCM-41}

A peneira molecular MCM-41 foi sintetizada pelo procedimento de tratamento hidrotérmico, baseada na patente de Silva, 2009. A síntese foi conduzida preparando uma solução aquosa, contendo a fonte de sílica (sílica aerosil 200) e de sódio (silicato de sódio), com agitação constante e temperatura compreendida entre 55 e $60^{\circ} \mathrm{C}$. A mistura resultante foi agitada até a completa homogeneidade por um período de 2 horas. Após este período, adicionou-se uma solução contendo o agente direcionador de estrutura brometo de cetiltrimetilamônio (CTMABr), a temperatura ambiente e sob agitação por um período de 1 hora.

O gel de composição molar: 1,0 CTMABr:4,0 SiO $2: 1 ~ \mathrm{Na}_{2} \mathrm{O}: 200 \mathrm{H}_{2} \mathrm{O}$, foi introduzido em um recipiente de teflon com tampa, inserido em uma autoclave de aço inox e aquecido a temperatura de $100^{\circ} \mathrm{C}$ em estufa, durante um período de 7 dias, controlando-se o $\mathrm{pH}$ do gel com ácido acético a cada 24 horas, mantendo-se na faixa de 9-10, até que o mesmo atingisse a estabilidade. O sólido final foi separado do sobrenadante por filtração, lavado com água deionizada e depois, seco em estufa.

\subsection{Caracterização}

Difração de raios $X(D R X)$ : Utilizou-se um difratômetro Shimadzu XRD-6000 com radiação $\mathrm{CuK} \alpha$, tensão de $40 \mathrm{KV}$, corrente de $30 \mathrm{~mA}$, tamanho do passo de $0,0202 \theta$ e tempo por passo de $1,000 \mathrm{~s}$, com velocidade de varredura de $2^{\circ}(2 \theta) / \mathrm{min}$, com ângulo $2 \theta$ percorrido de $1,5^{\circ}$ a $10^{\circ}$.

Adsorção física de nitrogênio (método de BET): Na obtenção das isotermas de adsorção e dessorção de nitrogênio a $-196^{\circ} \mathrm{C}$ utilizou-se um aparelho ASAP 2020 da Micromeritics.

\subsection{Remoção do Corante Azul Reativo}

Para avaliar o potencial da peneira molecular MCM-41 como adsorvente do corante Azul Reativo BF-5G, foram realizados os experimentos de banho finito variando o $\mathrm{pH}$ do meio 
reacional, que foi ajustado pela adição de ácido sulfúrico $0,1 \mathrm{M}$ e a massa do adsorvente (peneira molecular MCM-41).

Inicialmente foi preparada uma solução a $1000 \mathrm{mgL}^{-1}$ do corante azul reativo BF-5G, a partir da qual foram realizadas sucessivas diluições, cuja absorbâncias foram analisadas em um aparelho espectrofotométrico (espectrofotômetro UV- 1600 Pró-Análise), para obtenção da curva de calibração.

Diluiu-se a solução-mãe para uma concentração de $50 \mathrm{mgL}^{-1}$ e realizou-se ensaios em banho finito, sob agitação, à temperatura ambiente, em um shaker orbital, a $200 \mathrm{rpm}$ durante 24 horas. Em seguida as amostras foram filtradas objetivando a retirada da matéria sólida e o filtrado levado à análise espectrofotométrica visível, utilizando espectrofotômetro UV- 1600 Pró-Análise, para avaliar a quantidade de corante removido.

Para a realização dos experimentos foi utilizada a técnica do planejamento experimental, especificamente o planejamento fatorial $2^{2}$, com ponto central.

Os valores das variáveis do planejamento para os níveis (+) e (-), e para o ponto central, estão mostrados na Tabela 1.

Tabela 1- Valores escolhidos para os níveis (+), ( -) e (0).

\begin{tabular}{|c|c|c|c|}
\hline \multirow{2}{*}{ Variáveis } & \multicolumn{3}{|c|}{ Níveis } \\
\cline { 2 - 4 } & - & + & 0 \\
\hline $\begin{array}{c}\text { Quantidades de adsorvente } \\
(\mathrm{g} / 100 \mathrm{ml} \text { de solução) }\end{array}$ & 1,0 & 3,0 & 2,0 \\
\hline $\mathrm{pH}$ & 2 & 6 & 4 \\
\hline
\end{tabular}

A percentagem de remoção de corante foi calculada através da Equação (1):

$$
\% \operatorname{Re} m=\left(\frac{C_{0}-C_{e q}}{C_{0}}\right) * 100
$$

Em que, $C$ é a concentração final e $\mathrm{C}_{0}$ a concentração inicial da solução.

\section{RESULTADOS}

O resultado de difração de raios X da peneira molecular MCM-41 é apresentado na Figura 1. 


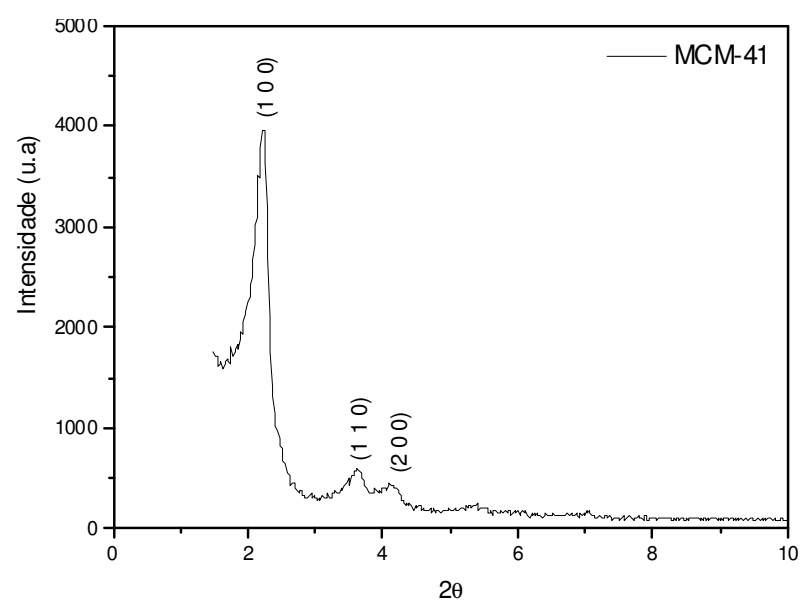

Figura 1 - Difratograma da MCM-41.

Os padrões de DRX da peneira molecular MCM-41 apresentou três picos característicos, um com elevada intensidade, atribuída à linha de reflexão do plano $\left(\begin{array}{lll}1 & 0 & 0\end{array}\right)$ e dois outros com menor intensidade, atribuídos às reflexões dos planos $\left(\begin{array}{lll}1 & 1 & 0\end{array}\right)$ e $\left(\begin{array}{lll}2 & 0 & 0\end{array}\right)$ característicos da estrutura hexagonal mesoporosa como descrito pelos pesquisadores da Mobil Oil Research and Development Co (Beck et al, 1992). Este padrão de difração indica uma estrutura hexagonal bem ordenada.

O espaçamento interplanar $\mathrm{d}(100)$ e o parâmetro unitário de célula $\left(\mathrm{a}_{0}\right)$ da peneira molecular mesoporosa foi determinado por meio das equações 2 e 3:

$$
\begin{gathered}
\mathrm{d}(100)=\lambda / 2(\operatorname{sen} \theta) \\
\mathrm{a}_{0}=2 \mathrm{~d}_{(100)} /(3)^{1 / 2}
\end{gathered}
$$

Em que, $\lambda$ o comprimento de onda da radiação incidente na amostra.

Tabela 2 - Espaço interplanar $\mathrm{d}_{100}$ e o parâmetro de cela unitária $\mathrm{a}_{0}$ das peneiras moleculares MCM-41.

\begin{tabular}{|c|c|c|c|}
\hline Parâmetro & $2 \theta$ & $\mathrm{d}(\mathrm{nm})$ & $\mathrm{a}_{0}(\mathrm{~nm})$ \\
\hline MCM-41 & 2,2 & 4,0 & 4,6 \\
\hline
\end{tabular}

O valor da distância interplanar e do parâmetro de cela unitário apresentado na Tabela 2 estão de acordo com a literatura. Tais parâmetros estão de acordo com os encontrados na literatura (Stucky et al., 1997; Silva, 2009).

A Figura 2 apresenta a isoterma de adsorção-dessorção de $\mathrm{N}_{2}$ a $-196{ }^{\circ} \mathrm{C}$ referente à amostra de MCM-41. 


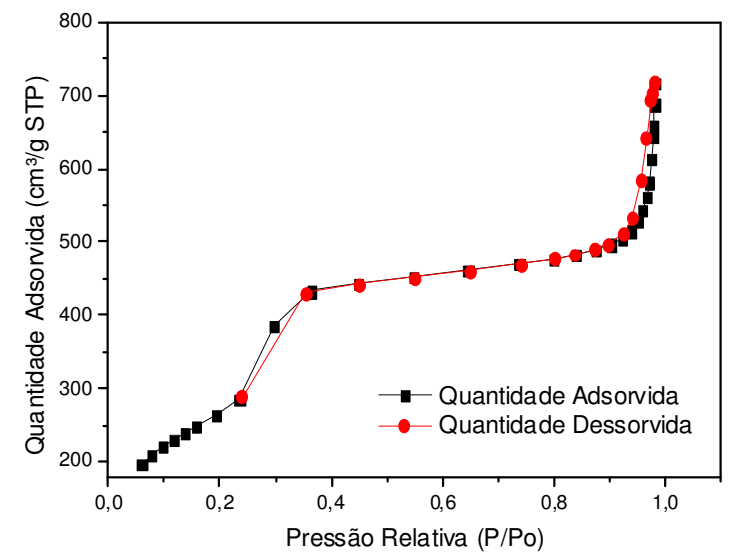

Figura 2 - Isoterma de adsorção-dessorção de $\mathrm{N}_{2}$ a $-196^{\circ} \mathrm{C}$ da peneira molecular MCM-41.

Pela análise da isoterma de adsorção-dessorção de $\mathrm{N}_{2}$ (Figura 2), é possível observar que elas são do tipo IV, que é característica de materiais mesoporosos altamente ordenados com estrutura hexagonal e uma larga distribuição de tamanho, quando se utiliza nitrogênio como adsorbato (Gaydhankar et al. 2006).

Nas isotermas apresentadas, observa-se três regiões: a primeira, dada a baixas pressões relativas $\left(\mathrm{P} / \mathrm{P}_{0}<0,2\right)$ corresponde à adsorção de $\mathrm{N}_{2}$ na monocamada, comum a todas as isotermas apresentadas; a segunda região dada entre $\mathrm{P} / \mathrm{P}_{0}=0,2-0,4$. Neste ponto, a quantidade de gás adsorvido aumenta abruptamente com pequena variação de pressão devido à condensação das moléculas do adsorbato abaixo de sua pressão de vapor em mesoporos primários (Biz et al, 1998). As isotermas apresentam histerese do tipo H1.

Segundo Leofanti et al., 1998, os histereses dos tipos H1, são comportamentos correspondes a materiais porosos constituídos por aglomerados rígidos de partículas de tamanho aproximadamente uniforme ordenadas regularmente. Correspondem também ao modelo de cilindros abertos nas duas extremidades. $\mathrm{E}$ a terceira $\left(\mathrm{P} / \mathrm{P}_{0}>0,9\right)$, comum a todas as isotermas, pode ser atribuída a adsorção das multicamadas da superfície externa (Schmidt et al, 1995), as curvas se tornam assintóticas, comportamento característico de condensação capilar em mesoporos secundários (Gregg et al., 1982; Ghosh et al., 2003).

Os resultados para a análise textural da peneira molecular MCM-41 estão apresentados na Tabela 3.

Tabela 3 - Análise textural da peneira molecular MCM-41.

\begin{tabular}{|c|c|c|c|}
\hline Amostra & $\mathrm{S}_{\mathrm{BET}}\left(\mathrm{m}^{2} / \mathrm{g}\right)$ & $\mathrm{V}_{\mathrm{p}}^{\text {total }}\left(\mathrm{cm}^{3} / \mathrm{g}\right)$ & $\mathrm{e}(\mathrm{nm})$ \\
\hline $\mathrm{MCM}-41$ & 927 & 1,28 & 1,6 \\
\hline
\end{tabular}

A peneira molecular MCM-41 apresentou alto valor de área superficial específica $927 \mathrm{~m}^{2} / \mathrm{g}$, e um volume total de poros $1,28 \mathrm{~cm}^{3} / \mathrm{g}$, estes valores estão de acordo com os encontrados na literatura (Gaydhankar et al., 2006).

Realizando uma combinação das técnicas, difração de raios $\mathrm{X}$ e adsorção física de $\mathrm{N}_{2}$, foi possível calcular a espessura da parede. A peneira molecular MCM-41 apresentou valor de 


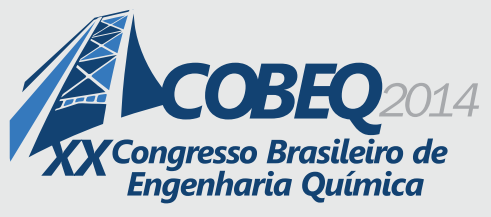

espessura de parede de 1,6 nm. Este valor é responsável pela estabilidade hidrotérmica da MCM41 e está próximo ao encontrado na literatura (Cano et al., 2010).

$\mathrm{Na}$ Tabela 4 os resultados de percentagem de remoção do corante azul reativo BF-5G pela peneira molecular MCM-41 são apresentados.

Tabela 4 - Resultados obtidos do planejamento fatorial $2^{2}$ para o corante Azul Reativo BF-5G.

\begin{tabular}{|c|c|c|c|c|}
\hline Ensaios & $\begin{array}{c}\text { Quantidade de adsorvente } \\
(\mathrm{g}) / 100 \mathrm{ml} \text { de solução }\end{array}$ & $\mathrm{pH}$ & \%Remoção & qeq (mg/g) \\
\hline 1 & 0,25 & 2 & 90 & 4,49 \\
\hline 2 & 0,25 & 6 & 38 & 1,90 \\
\hline 3 & 0,75 & 2 & 56 & 0,94 \\
\hline 4 & 0,75 & 6 & 99 & 1,65 \\
\hline 5 & 0,5 & 4 & 97 & 2,43 \\
\hline 6 & 0,5 & 4 & 96 & 2,41 \\
\hline 7 & 0,5 & 4 & 98 & 2,45 \\
\hline
\end{tabular}

A interpretação dos resultados apresentados na Tabela 4 conduz aos seguintes resultados:

a) A melhor percentagem de remoção total de corante azul reativo foi no ensaio 4 (99\%), no qual o pH é 6 e a quantidade de adsorvente utilizada é $0,75 \mathrm{~g} / 100 \mathrm{ml}$ de solução;

b) No geral, as remoções são consideradas satisfatórias, já que apenas dois ensaios tiveram remoções abaixo de $90 \%$.

c) Verificou-se também que o melhor resultado levando em consideração a capacidade de remoção do corante azul reativo foi no ensaio $1(4,49 \mathrm{mg} / \mathrm{g})$, onde o pH é 2 e a quantidade de adsorvente é $0,25 \mathrm{~g} / \mathrm{ml}$ de solução.

A influência das variáveis, quantidade de adsorvente e $\mathrm{pH}$, sobre a capacidade e a percentagem de remoção de corantes pode ser observada através dos gráficos de pareto, Figuras 3(a) e 3(b), onde as variáveis apresentam uma influência estatisticamente significativa, quando seu efeito ultrapassa a linha de $\mathrm{p}=0,05$.
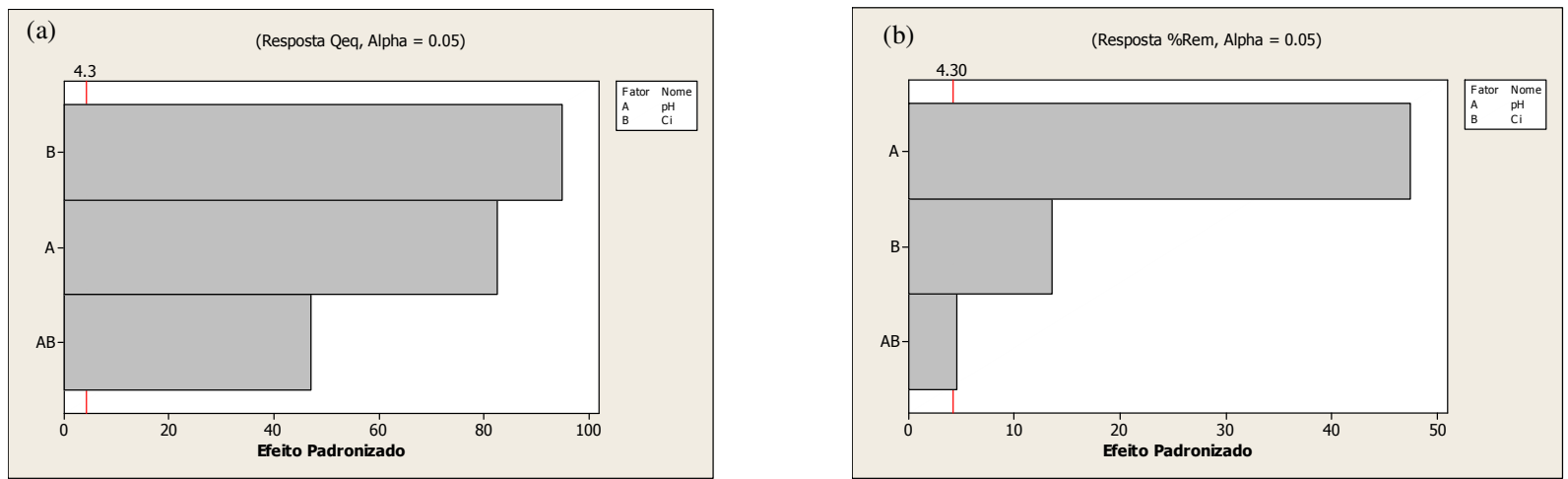

Figura 3 - Gráfico de Pareto para (a) capacidade de remoção e (b) percentagem de remoção do corante Azul reativo BF-5G.. 


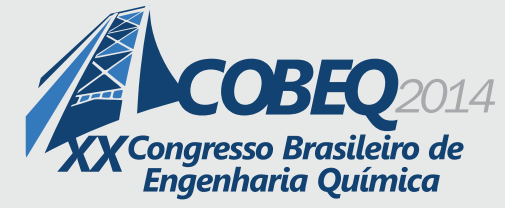

Para a capacidade de remoção, o gráfico de pareto apresentado na Figura 3(a), mostra que os três parâmetros avaliados tiveram efeito significativo sobre a resposta ao nível de confiança de $95 \%$, o pH, a massa de adsorvente utilizada e a interação entre o pH e a massa.

O resultado avaliado levando em consideração a percentagem de remoção apresentada na Figura 3(b) mostra que todas as variáveis estudadas são significativas ao nível de $95 \%$ de confiança bem como a interação entre eles.

\section{CONCLUSÕES}

Os padrões de difração de raios X apresentaram picos característicos da estrutura MCM-41, evidenciando formação da estrutura bem definida de mesoporos. Pela análise textural da peneira molecular MCM-41 foi verificada elevada área superficial específica e dimensões de poro de acordo com a literatura. Os resultados de percentagem de remoção e capacidade de remoção do corante azul reativo em sistema de banho finito, apresentaram valores bastante satisfatórios para a peneira molecular mesoporosa MCM-41, com valor máximo de remoção de $99 \%$ no ensaio 4 e capacidade de remoção com valor máximo de $4,49 \mathrm{mg} / \mathrm{g}$ no ensaio 1. A partir da análise estatística, conclui-se que as respostas percentagem de remoção de corante azul reativo (\%Rem) e capacidade de remoção (qeq) são influenciadas significativamente pelos fatores analisados (quantidade de adsorvente e $\mathrm{pH}$ ).

\section{REFERÊNCIAS}

BECK, J.S., VARTULI, J.C., ROTH, W.J., LEONOWICZ, M.E., KRESGE, C.T., SCHMITT, K.D., CHU, C.T.-W., OLSON, D. H., SHEPPARD, E.W., MCCULLEN, S.B., HIGGINS, J. B.; SCHLENKER, J. L., A new family of mesoporous molecular sieves prepared liquid crystal templates. J. Am. Chem. Soc., v. 114, p. 10834-10843, 1992.

BIZ, S.; OCCELLI, M. Synthesis and Characterization of mesostructuresd Materials. Catal. Rev Sci. Eng., v. 40, p. 329-335, 1998.

CANO, L. A.; CAGNOli, M. V.; FELlENZ, N. A., BENGOA J. F.; GALlEGOS N. G.; ALVAREZ, A. M.; MARCHETTI, S. G. Fischer-Tropsch synthesis. Influence of the crystal size of iron active species on the activity and selectivity. Appl. Catal. A: General, v. 379, p. 105-110, 2010.

CHAVES, J.R.; ROLIM, H. O.; SILVA FILHO, H. A.; SANTOS, E. V. M. Remoção do corante Azul de Metileno (AM) utilizando como adsorvente a cinza da casca do arroz (CCA). In: $26^{\circ}$ CONGRESSO BRASILEIRO DE ENGENHARIA SANITÁRIA E AMBIENTAL. Porto Alegre RS: FIERGS, 2011. 
GAYDHANKAR, T. R.; SAMUEL, V.; DAN JOSHI, P. N. Hydrothermal Synthesis of MCM-41 Using Differently Manufactured Amorphous Dioxosilicon Sources. Mater. Lett., v. 60, p. 957961, 2006.

GHOSH, A.; PATRA, C.R.; MUKHERJEE, P.; KUMAR, R.; SASTRY, M. Preparation and stabilization of gold nanoparticles formed by in situ reduction of aqueous chloroaurate ions within surface-modified mesoporous silica. Microporous Mesoporous Mater., v. 58, p. 201-211, 2003.

GREGG S. J.; SING K.S.W. Adsorption, Surface and Porosity. $2^{\mathrm{a}}$ ed. Academic Press; London, UK: 1982.

GUARATINI, C.I.; ZANONI, M.V. Corantes Têxteis. Química Nova., v. 23, p. 71-78, 2000.

LEOFANTI, G.; PADOVAN, M.; TOZZOLA, G.; VENTURELLI, B. Surface area and pore texture of catalysts. Catal. Today, v. 41, p. 207-219, 1998.

SILVA, M.L.P. Síntese e caracterização de peneiras moleculares mesoporosas do tipo MCM-41 e AlMCM-41 a partir de fontes alternativas de sílica e de alumínio. 63f. Tese (doutorado em Engenharia de Materiais) Universidade do Rio Grande do Norte - Natal, 2009.

OLIVEIRA, T. F.; BEZERRA, N. S. S.; LEITÃO, N. M. C.; SILVA, M. L. P; GOMES, K. K. P., Remoção do Corante Vermelho Reativo por Argila Esmectita Em Sistema de Banho Finito: Influência do $\mathrm{pH}$ e da Quantidade de Adsorvente. in Anais do $9^{\circ}$ Encontro Brasileiro sobre Adsorção, 2012.

RYOO, R.; HIM, J.M.; KO, C.H. Improvement of structural integrity of mesoporous molecular sieves for practical applications. Stud. Surface Sci. Catal., v. 117, p. 151-158, 1998.

STUCKY, G.D.; HUO, Q.; FIROUZI, A.; CHMELKA, B.F.; SCHACHT, S.; VOIGT-MARTIN, I.G.; SCHÜTH, F. Directed synthesis of organic / inorganic composite structures. Stud. Surface Sci. Catal., v. 105, p. 3-28, 1997.

VIMONSES, V.; JIN, B.; CHOW, C. W. K.; SAINT, C. Enhancing removal efficiency of anionic dye by combination and calcination of clay materials and calcium hydroxide. J. Hazard. Mater., 171, p. 941-947, 2009. 